\title{
IMPORTANCIA DE LA INTERVENCIÓN DE LA ADMINISTRACIÓN EN EL PROCESO DE TRANSICIÓN A NIIF EN LAS PYMES DE SOGAMOSO, BOYACÁ
}

\section{IMPORTANCE OF THE INTERVENTION OF THE ADMINISTRATION IN THE TRANSITION PROCESS TO INTERNATIONAL FINANCIAL REPORTING STANDARDS “IFRS" IN THE MICRO-ENTERPRISES IN SOGAMOSO, BOYACÁ}

\author{
Derly Yasmin Pineda Calixto
}

\begin{abstract}
Resumen
Se tiene la concepción que el proceso de convergencia a Normas Internacionales de Información Financiera, NIIF, le concierne únicamente a los profesionales de contabilidad y al área financiera de las empresas, cuando en realidad és un proceso que involucra a la gerencia y debe iniciarse desde sus más altos niveles de administración empresarial, debido a la importancia y trascendencia de dicho proyecto que afecta a toda la organización, incluso su estrategia de negocio. La administración de las PyMES de Sogamoso no está exenta de las consideraciones anteriores, pues no se han detenido a reflexionar sobre los impactos que el proceso de convergencia trae a la organización y la incidencia en la toma de decisiones, al igual que las ventajas competitivas que el mejoramiento en la calidad de la información financiera puede traerles. Este escrito pretende mediante un enfoque exploratorio analítico, visualizar la importancia de la intervención de la administración en el proceso de transición a NIIF. Las conclusiones de este documento están encaminadas a proponer estrategias que orienten y permitan una mejor intervención de la gerencia en el proceso de convergencia, especialmente en las PyMES de Sogamoso.
\end{abstract}

Palabras clave: convergencia, PyMES, competitividad, NIIF, administración.
Recibido: Enero 20 de 2013

Abstract

Aceptado: Junio 20 de 2013

There is the notion that the rationale of the convergence to the International Financial Reporting Standards (IFRS) merely governs professionals in accounting and finance departments of their respective entities. The fact is that it is also a process that involves all levels of management, and should generally commence from the highest levels of management, due to the importance and meaning of this project that affects the entire organization, including but not limited to its business strategies. The management of micro-enterprises in Sogamoso is also not an exempt. In a way, it hasn't considered the impact that the process of convergence brings to the organization, and its impact in decision-making, as well as the competitive advantages that the improvement in the quality of the financial information can bring them. This article's aim is to cover this subject through an exploratory analytical approach, and to visualize the importance of the intervention of the administration in the process of transition to the IFRS. The conclusions of this document are proposing strategies that guide and allow a better management intervention in the convergence process specially in Sogamoso's microenterprises.

Key words: convergence, SMEs, competitiveness, IFRS, management. 


\section{Introducción}

El fenómeno económico llamado globalización, los nuevos retos y desafíos que aparecen en la dinámica de las organizaciones y la constante actividad de carácter económico y administrativo que viven las empresas hacen que aquellas que pretendan permanecer en el tiempo y en los mercados deban acatar y atender las normatividades que regulan sus actividades. En la internacionalización de los mercados económicos, la necesidad de seguir políticas de buen gobierno y en la generalidad de las exigencias de los negocios, los estándares internacionales juegan un papel fundamental tanto en la preparación de información financiera como en el monitoreo y la aplicación de nuevos modelos de administración (Janica \& Piñero, 2004).

En el ámbito mundial la comunidad económica ha proferido una regulación que pretende uniformar la información y es así como aparecen las Normas Internacionales de Información Financiera, NIIF; además, Colombia ha entrado en el proceso de transición a dichos estándares y ha emitido las leyes y decretos respectivos para tal fin. Por tal razón las organizaciones deben cumplir con esta reglamentación, buscar estrategias que les permitan realizar el proceso de convergencia de la mejor manera y satisfacer los requerimientos que se les presentan; es ahí donde la gerencia juega un papel fundamental al intervenir, participar y gestionar el proceso de transición a NIIF.

Sin embargo, este proceso no ha logrado la suficiente atención ni intervención de los administradores y se considera como un asunto que atañe solamente al departamento de contabilidad de la empresa, sin tener en cuenta los cambios que impactan la organización. Adquiere importancia entonces el estudio de cómo las empresas y sus administradores pueden llegar a enfrentar los cambios referentes a la contabilidad con el fin de adquirir ventajas competitivas. Cambios como el de la conversión a NIIF cuya finalidad es expedir reglamentación contable que conforme un sistema único y homogéneo de alta calidad, lleva a que los informes financieros brinden información transparente, comparable y confiable útil para la toma de decisiones de las partes interesadas; así se podrá mejorar la productividad de la actividad empresarial, permitiéndoles de esta forma ser competitivos en mercados globalizados, con el propósito de apoyar la internacionalización de la economía.

Los administradores de las Pymes en Sogamoso, deben ser consientes de las consideraciones anteriores y prepararse para su participación en dicho proceso. Si se tiene en cuenta que estas empresas no poseen herramientas ni recursos suficientes para enfrentar estos cambios, se hace necesario plantear estrategias de mejoramiento que permitan, mediante la intervención de la administración, optimizar el proceso de transición a NIIF.

\section{Origen y evolución de las NIIF}

Para entender la exigencia de la implementación de las NIIF y para conocer su aplicación en las empresas de Sogamoso, Boyacá, es importante hablar acerca de su origen. Las crisis económicas, financieras y cambiarias de mediados de los 90, en especial la de México y Asia Oriental, impactaron las economías emergentes. Esto incidió en que la comunidad internacional planteara la necesidad de fortalecer, modificar o reconstruir la Arquitectura Financiera Internacional, AFI, vigente, a través de una Nueva Arquitectura Financiera Internacional, NAFI, con el fin de proteger los intereses de los proveedores de capital, en procura de minimizar el riesgo y disminuir costos dentro de la actividad empresarial (Bustelo, 2005). Estas crisis se dieron como consecuencia de la integración económica a través de la liberalización generalizada de las transacciones comerciales, a la desregularización de los mercados financieros domésticos y al incremento de los flujos internacionales de capital, fenómenos que alteraron el Sistema Financiero 
Internacional (Castiglione, 2012).

La NAFI está bajo la coordinación del G20, cuya función es la construcción de un marco de regulación más estricto y globalmente consistente, con el fin de reducir la probabilidad de ocurrencia de una nueva crisis financiera, con miras a asentar e incrementar el crecimiento económico a escala mundial (Carrera, 2009).

\subsection{Estandarización de la regulación contable}

La debilidad de los sistemas financieros de las economías emergentes se debe a una inadecuada regulación y ausencia de sistemas de control de la gestión de los agentes; por ello uno de los objetivos de la NAFI es el de fortificar los sistemas financieros domésticos de estas economías. De manera que es primordial el adelanto y la adopción efectiva de estándares internacionales en materia de contabilidad y de conducta de gestión corporativa, donde evidentemente, no es suficiente con desarrollar estos estándares sino que es necesario también asegurar su adopción generalizada y cumplimiento efectivo (Ramos, 2001).

"Los estándares no son imposiciones de los países desarrollados, son simplemente un conjunto de principios que desarrollan las mejores prácticas en un tema específico relacionado con la estabilidad financiera y la transparencia en los mercados" (Varón, 2012, p.17). Dichos estándares están basados en principios ya que las normas son discrecionales y de libre interpretación.

Pero como en todo proceso de cambio, existen opositores que critican la estandarización de la regulación contable, como Mejía, Montes \& Montilla (2008, p. 80), quienes afirman que: "La aceptación de los estándares internacionales de la disciplina contable es una entrega de la autonomía en materia de regulación nacional, endosa la potestad de las naciones de auto regularse y entrega a los organismos internacionales la autoridad de normalizar el ejercicio profesional. Constituye una renuncia de cada país a construir su modelo de desarrollo, no sólo en materia contable, sino en los campos económico, social y político".

Otros autores como García \& Sierra (2012) plantean que la norma no es neutral ya que trae beneficios para unos agentes y pérdidas para otros. Quienes se afectan con dicha adopción serían los estados, porque entregan la soberanía de la regulación contable; los profesionales de la contabilidad, porque debe asumir los precios de entrenamiento y las empresas privadas ya que tienen que asumir el costo del cambio de regulación, entre otros.

A pesar de las consideraciones anteriores, el proceso de convergencia a NIIF avanza y se ha convertido en un asunto de carácter obligatorio, exigencia establecida en los últimos años, ya que a lo largo de la historia, cada país ha sido libre para adoptar las prácticas contables de otros países, acomodándolas a sus características locales. Pero solo ha sido durante el último tercio del siglo $X X$ cuando se han visto intentos para armonizar la regulación contable (Wyman, 1990, p.13).

El proceso de convergencia internacional comenzó en 1973, cuando 16 órganos profesionales de contabilidad, acordaron formar la IASC que, en 2001, se convirtió en la IASB. La IASB elabora estándares mundiales que se conocen globalmente como NIIF (UNCTAD, 2005). En Colombia existe legislación que exige para los años 2015 y 2016 a las organizaciones del ámbito nacional tener su información contable conforme a los estándares internacionales. En el año 2009 fue expedida la ley 1314 y en años posteriores, algunos decretos que igualmente buscan la aplicación de las NIIF, sustituyendo el actual marco conceptual de la contabilidad decreto 2649.

Una vez se inició el proceso de convergencia, un número sin precedentes de empresas y países de todo el mundo adoptaron las NIIF como base para elaborar los estados financieros. Esto se da, en 
primer lugar, por las aspiraciones de NIIF que pretenden brindar información financiera de alta calidad y transparencia, que permite la comparabilidad, requerida por los usuarios, de la información para la toma de decisiones, lo que conlleva a disminuir la incertidumbre que afecta el costo de sus negociaciones (Ramírez \& Suárez, 2010). En segundo lugar, por la percepción de los individuos directamente relacionados con las actividades de contabilidad y auditoría el cual es positivo, gracias a las ventajas que se obtienen en la aplicación de NIIF, estas ventajas pueden ser vistas en la comparabilidad de la información contable en el ámbito mundial y la reducción de actividades ilegales, especialmente los fraudes en la preparación y presentación de estados financieros (Bozkurt, Islamoglu \& Oz, 2013).

También se ha señalado que las diferentes propuestas regulatorias suponen una buena oportunidad para tratar de recuperar la confianza de los inversores, darle mayor confiabilidad a la información contable provista por las empresas para sus inversionistas y para la comunidad de negocios en general, lo que conlleva a disminuir el riesgo de nuevas crisis económicas. Por ende la presentación de información contable, bajo los estándares internacionales, mejora la competitividad y productividad de la organización permitiéndole de esta manera penetrar en mercados globalizados, lo que genera un impulso para el comercio internacional, gracias a la eliminación de los obstáculos correspondientes a las diferentes prácticas nacionales. (Saizarbitoria \& Casadesús, 2006).

\subsection{Internacionalización de las organizaciones}

El surgimiento de los acelerados procesos de internacionalización de las economías ha creado la necesidad de que las empresas se enfrenten a este desafío, realicen cambios y se adapten a ellos de manera rápida con el fin de mantenerse en el mercado. Según Bassino (2009, p. 4): “Durante las últimas dos décadas, la globalización de la economía mundial tuvo una clara incidencia en la forma de realizar negocios, de acceder a fuentes de financiación, en las políticas de recursos humanos, en las normas tributarias y también en la aplicación de un lenguaje común para interpretar los estados contables en todos los mercados".

Sur América no está libre de las consideraciones anteriores, "los países latinoamericanos han sometido sus economías a los imperativos de la globalización siguiendo distintas vías, ritmos y mecanismos" (Arango \& López, 1999). Colombia no está exenta de los efectos de la globalización de la economía mundial, ni puede permanecer aislada de sus tendencias (Calderón, Naranjo \& Álvarez, 2007); esa es una de las razones que han conducido a la firma de tratados de libre comercio porque son un instrumento útil para integrar al país en las corrientes internacionales del comercio (CTCP, 2012a).

Sin embargo, las empresas de Boyacá, en su gran mayoría no cuentan con herramientas de planeación estratégica que les permitan hacer frente a los retos que traen consigo la globalización de los mercados; el empresario boyacense se caracteriza por la gestión de su actividades en corto plazo sin llegar a establecer un plan concreto que logre involucrar a la empresa en los mercados a largo plazo afectando de gran manera su competitividad (Ávila, 2013). Esto lo confirma Zambrano (2012, p. 190), "los niveles de competitividad en el municipio de Sogamoso demuestran que existe un alto grado de inconformismo por parte de los gerentes y dueños de empresas".

\section{Las PyMES y la gestión de la administración}

\subsection{Contexto internacional y regional de las PyMES}

Las pequeñas y medianas empresas desempeñan un papel importante en la vida económica y social de la mayoría de los países, siendo estas la columna vertebral de su economía (Rodríguez, 2008). Las PyMES son uno de los pilares 
fundamentales de las economías modernas debido a su gran capacidad de generación de riqueza, empleo e importante participación en el desarrollo económico, principalmente de las economías emergentes (Rodeiro \& López, 2007). "La dinámica de los países latinoamericanos evidencia la notoria importancia de las PyMES dentro de sus estructuras productivas, pues realizan aportes significativos en términos de producción, generación de empleo y de impacto social" según Correa y Londoño (2009), citado por (Yandar, 2011, p. 1).

Para el caso colombiano las PyMES agrupan cerca del $96 \%$ de las empresas, participan con un $30 \%$ del total de la producción, aportan un poco más del $70 \%$ del empleo, remiten un poco más del $30 \%$ de las exportaciones y generan alrededor del 50\% de los salarios. Así mismo contribuyen al $41 \%$ del PIB nacional (Mondragón, 2011, p. 12). Como se puede evidenciar ,"Ias PyMES se han consolidado como foco de desarrollo y elemento clave para el crecimiento económico no solo porque configuran un alto porcentaje del tejido empresarial colombiano, sino por su aporte al desarrollo de la economía del país y en la disminución de la pobreza"(Vélez, 2009, p. 1).

Sogamoso no está exenta de las consideraciones anteriores, desde los años 50 el desarrollo de esta ciudad ha sido apalancado por empresas como: Acerías Paz de Río, Cementos Boyacá (hoy Holcim), Cementos Paz de Río (hoy Argos), entre otras, las cuales hacen que la generación de empleo se vea influenciada por este sector. La ubicación cercana de grandes yacimientos de minerales, que sirven de materia prima, hace que alrededor de las industrias aparezcan zonas como el parque industrial y las proyectadas zonas francas para el departamento; junto con estas aparece un buen número de pequeñas y medianas empresas que contribuyen al fortalecimiento y crecimiento económico de la ciudad.

De ahí la importancia de generar estrategias que les permitan a las PyMES de Sogamoso ser más eficientes, productivas y competitivas no tan solo en el ámbito nacional sino internacional, incrementando a la vez el interés por las exportaciones. Es importante que estas empresas creen una infraestructura contable que sea coherente con las exigencias de las entidades extranjeras, la información contable se debe adecuar a la evolución de otros países (Burgos, 2007). Sin embargo existen suficientes hallazgos de la mediana capacidad de liderazgo de los empresarios de las PyMES, en la nueva sociedad de la información y el conocimiento y poca visión prospectiva en las variables analizadas, para enfrentar oportunamente los procesos competitivos de globalización (Castells, 2000) citado por (Quiroga, 2007, p. 33).

En este sentido, los administradores están obligados a plantear nuevas estrategias que permitan conservar o incrementar el nivel de competitividad de sus empresas, como la de elaborar estados contables normalizados que puedan ser universalmente entendidos e interpretados, de tal forma que orienten la oportuna asignación de los recursos disponibles, ya que los inversores elegirán aquellos proyectos de inversión de las empresas más competitivas, que deben emitir información relevante y transparente sobre su pasado, presente y futuro (Evia, 2006; Mallo \& Pulido, 2005; Zapata, 2001).

De tal manera que para enfrentar oportunamente los procesos de la globalización es fundamental asumir actuaciones que les admita mantener sus organizaciones en el mercado, de ahí la importancia de la convergencia a estándares internacionales en donde la adopción de NIIF para PyMES es un elemento primordial para el alcance de este propósito.

\subsection{NIIF para PyMES}

Las NIIF fueron elaboradas tomando en consideración inversores internacionales que deben analizar información proveniente de estados financieros de empresas que cotizan en 
bolsas en todo el mundo, buscando oportunidades de inversiones más seguras y a más bajo precio. Las empresas emisoras de valores, en número, constituyen el $10 \%$ de las empresas mundialmente establecidas, pero representan el $90 \%$ del PIB mundial. Una debilidad de las NIIF era la falta de normas menos complejas para que pudieran ser aplicadas por el $90 \%$ restante de las empresas, las denominadas Pequeñas y Medianas Empresas (PyMES). Finalmente, el 9 de julio de 2009 el IASB emitió las NIIF para PyMES (Bassino, 2009 , p. 5). Cerca de 70 jurisdicciones de todo el mundo ya han adoptado las NIIF para PyMES o públicamente han señalado o propuesto un plan para adoptarlo en los próximos tres años (Mantilla, 2012), según consideraciones del vicepresidente Latinoamericano de Emisores de Normas de Información Financiera (GLENIF), (Gil, 2013).

Después de esta amplia adopción internacional de las NIIF, se ha hecho evidente que las distintas partes interesadas, siguen encontrando problemas prácticos de aplicación. Estos desafíos se refieren normalmente a los temas de desarrollo institucional, la ejecución y la capacidad de ejecución técnica. Las PyMES tienen una norma internacional específica, que es más pequeña, las PyMES no tendrán una norma sustancialmente más sencilla, porque esas disposiciones provienen conceptualmente de las NIIF completas. No obstante, la PyME va a tener menor cantidad de normas para estudiar, pero los pasos de implementación son básicamente los mismos.

Según Mejía, Montes \& Montilla (2008), existen algunos obstáculos en el proceso de transición, los países que han adoptado los estándares internacionales de contabilidad sin ningún tipo de reflexión y debate se han encontrado con obstáculos en ocasiones insalvables, ejemplo de estos son: Fecha de vigencia de aplicación de los estándares internacionales, los cambios en muy poco tiempo dejan obsoleta la regulación aprobada frente las nuevas regulaciones internacionales; escasa comunicación entre las personas interesadas en el proyecto; traducción de los estándares internacionales, en ocasiones, no se ha terminado de traducir el borrador cuando un nuevo borrador o estándar es emitido de forma oficial; complejidad y estructura de los estándares internacionales, obligan a que su aplicación sea coordinada por expertos en el tema; cambios constantes en los estándares internacionales de contabilidad y auditoría; los cambios que se presentan son frecuentes, implican volúmenes importantes de material e insertan nuevas técnicas y hacen más complejo el proceso de formación de expertos en estándares internacionales y la formación de personal experto en estándares internacionales de contaduría, los profesionales expertos en el tema, son muy pocos frente al desafío que representa.

Este panorama internacional lleva a reflexionar sobre aspectos como: si en los países desarrollados, que manejan la economía mundial a su libre albedrío, se presentan problemas en términos de la estandarización y regulación contable internacional, ¿qué podemos seguir esperando en Colombia, que apenas está reflexionando sobre la conveniencia de adaptar estándares internacionales? (Cano, 2009). Sin embargo las empresas colombianas tienen ventaja de conocer el efecto de la aplicación de NIIF en países que ya se han sometido al proceso de convergencia, contando con la fortaleza del efecto experiencia (Saizarbitoria \& Casadesús, 2006). Según el CTCP (2011), La conversión a estándares internacionales en Europa, la magnitud y complejidad del proyecto y el tiempo requerido fueron subestimados, inicialmente el proyecto careció de una aceptación adecuada de la alta gerencia, y los proyectos sufrieron de una administración pobre, esta es una de las razones que hace que el abordaje de esta temática se tenga que hacer desde los cuadros directivos y gerenciales de las empresas y se convierta en factor fundamental para el desarrollo y sostenimiento de las mismas. Basado en la experiencia de otros países, una prioridad inmediata para la administración es contar con recursos humanos entrenados, que tengan 
conocimientos y habilidades relacionados con la norma internacional, siendo este un reto significativo que las empresas deberán contemplar.

Según Maticorena (2013, p. 1): El reto para las PyMES es 20 veces más grande que para las empresas de mayor tamaño debido a que quizás no cuentan con suficientes recursos económicos Para evitar el caos, la solución está en lograr que las PyMES se concienticen de la urgencia de iniciar su proceso de conversión en el año 2013, por lo menos a través de la capacitación y la elaboración de un diagnóstico que identifique los impactos de la conversión en su empresa. De no hacerlo en el 2013, la PyME en Colombia corre el riesgo de no encontrar en el 2014 dónde capacitarse o de no encontrar un consultor que lo guíe en este importante esfuerzo.

Impactos que se verán reflejados en la gran mayoría de las áreas de la organización ya que, cualquier función del negocio requerida para preparar información financiera o impactada por información financiera, tiene el potencial para ser afectada por el cambio, para algunas áreas, los efectos serán grandes, para otras, serán menores, algunas implicaciones, pueden ser: Sistemas de tecnología de la información, planes de compensación para ejecutivos y empleados, impuestos, razones y convenios financieros, controles internos y procesos, reporte gerencial entre otros. De manera que la información recibida en el área contable es el resultado de una serie de procesos empresariales, allí se recibe su efecto y se registran, las NIIF pretenden que desde que nace la transacción hasta que termine se intercambie información de calidad, en cada adelanto de este proceso existen participantes de diferentes áreas, que por ende se van a ver impactadas por la nueva regulación (Flórez, 2012).

\subsection{La administración y el área contable}

Cuando se habla de convergencia a NIIF se tiene la concepción equivocada de que se trata de un simple ejercicio técnico contable, este proceso implicará un ejercicio de cambio gerencial del negocio y debe ser enfocado utilizando una metodología estructurada basada en las mejores prácticas de administración (CTCP, 2012b). De conformidad con lo establecido en el marco conceptual de NIIF, la responsabilidad principal por la preparación y presentación de los estados financieros corresponde a la gerencia de la entidad, refiriéndose a la función gerencial como tal y no a un cargo determinado, es decir esta puede ser ejercida por un presidente un gerente general, representante legal etc., por consiguiente el proceso de implementación a NIIF no es propia de los contadores Públicos. (Colegio de Peritos Mercantiles y Contadores Públicos de Honduras, 2011).

La ley 222, Artículo 37 (1995), obliga a los administradores a certificar conjuntamente con el contador la verificación de las afirmaciones contenidas en los estados financieros. Sin embargo, en Colombia la convergencia hacia las NIIF, pareciera que atañe únicamente a los profesionales de la Contaduría Pública, dada su amplia participación en los diferentes foros, orientados a la socialización y sensibilización de estándares (Londoño, 2011).

Este proceso tiene que formarse a partir de la administración, de acuerdo con la circular externa 115-000002 I (2012, p. 4), expedida por la Superintendencia de Sociedades, la cual considera: "que el cambio que conlleva la conversión a NIIF tiene que iniciarse desde los más altos niveles de administración empresarial (representante legal, junta directiva, gerentes) pues es allí donde tienen origen las principales decisiones relacionadas con políticas contables y financieras". Así, la implementación de las NIIF debe asumirse como un desafío general de las organizaciones, procurando la capacitación del personal directivo para que desde allí se puedan direccionar los procesos que permitan tener la información organizacional acorde con esa normatividad. 
En reuniones con empresarios de Sogamoso, ellos manifiestan su preocupación reconociendo que no tienen conocimiento, ni personal capacitado para enfrentar los nuevos retos que aparecen para ellos en torno a esta temática. Según González, Rodríguez \& Duarte (2011), las PyMES de Sogamoso, no cuentan con recursos económicos, herramientas gerenciales y acceso a consultoría que les permitan aumentar su productividad, y por ende su competitividad en los mercados actuales. En efecto, las empresas boyacenses presentan atrasos significativos en sistemas de gestión e innovación, y sus principales deficiencias están centradas en las áreas de planeación y procesos administrativos, lo cual afecta su capacidad competitiva (Bermúdez \& Gutiérrez, 2012).

Las estimaciones anteriores se reflejan en la baja participación e importancia que asumen los administradores de las PyMES de Sogamoso, con respecto al proceso de transición a NIIF. En éste sentido, Contreras (2010) afirma que la implementación y aplicación de las NIIF para PyMES ocasiona una inversión en tiempo, recursos humanos y económicos, que son relevantes para las empresas. Por ello se hace necesario diseñar y presentar para su aplicación, estrategias que permitan un mejor abordaje de la gerencia al proceso de convergencia a NIIF, buscando que se amplíen los panoramas competitivos y se minimice el efecto causado por el cambio.

La gerencia de las PyMES de Sogamoso debe tomar consciencia de los beneficios de reportar un conjunto completo de estados financieros con base en estándares contables internacionales, que facilitará el acceso a los mercados y la posible reducción de costos para las empresas y, por lo tanto, el mejoramiento de la competitividad. Lo anterior dado que la generación de ventajas competitivas, por parte de la empresa boyacense, está condicionada en gran parte por las personas que conforman la organización y por sus iniciativas en la nueva formulación de alternativas empresariales (González, Cerón \& Alcazar, 2010). Esto es más preocupante cuando los administradores de dichas empresas se identifican por gestionar planes a corto plazo y por no contar con herramientas de planeación estratégica efectiva, afectando de manera importante su competitividad (Ávila, 2013).

A través de conversaciones con empresarios de las PyMES de Sogamoso, en el marco proporcionado por la consultoría de gestión en innovación ofrecido por el convenio entre la Cámara de Comercio de Sogamoso y la Cámara de Comercio de Bucaramanga, se evidencia que dichas empresas enfrentan varios problemas. Uno de ellos es que no se tiene una cultura del mercadeo, es decir el uso de variables administrativas y operativas asociadas con la efectividad; así, los empresarios de las PyMES enfrentan fuertes dificultades, en lo referente a los entornos macro y microeconómico, que fueron consignadas por ellos como amenazas. Estas consideraciones se fortalecen ya que según Zambrano (2012), existe un alto grado de inconformismo en el tema de competitividad por parte de los administradores y empresarios de la ciudad.

Se puede decir que el componente administrativo de la Gestión de las PyMES del sector industrial de Sogamoso requiere de una regeneración y la disposición, no solo de actitud sino de conocimientos, que permitan mejorar la actividad y orientación empresarial hacia niveles que las ubiquen, al menos, dentro de los rangos de posibilidades de permanencia y proyección a estados más generadores de rendimientos. Los resultados muestran que las PyMES consideradas está, en su mayoría, en una etapa de despegue o desarrollo, con brechas importantes en: gestión, procesos, bienes o servicios y mercado de la innovación (Gutiérrez \& Bermúdez, 2011).

Es importante que la dirección de la empresa dentro de las estrategias para mejorar la competitividad, enfoque la innovación y adaptación a los cambios acelerados del mercado, como un proceso estratégico y desde una perspectiva global, (Cuervo 2003) citado por 
(Rodeiro \& López, 2007), estrategias que deben incluir el proceso de convergencia a NIIF como instrumento fundamental para mejorar la competitividad de la organización frente a los desafíos de la globalización.

En su mayoría, las investigaciones realizadas sobre las organizaciones se han enfocado en elementos tales como la estructura, el tamaño de la organización y su diseño (Bedodo \& Giglio, 2006). En éste sentido, adquiere importancia el estudio de cómo las empresas enfrentan los cambios referentes a la contabilidad con el fin de adquirir ventajas competitivas. Los administradores deben basar la toma de decisiones gerenciales en el análisis de las distintas herramientas de control de gestión, como son los estados financieros, de manera que, es fundamental tener claro los resultados secundarios que se pueden generar, dependiendo de la calidad de la información, aspectos que responden al llamado de las nuevas perspectivas financieras globalizadas (Carreño, 2012).

Se observa que aún cuando se acepta que la contabilidad es un instrumento valioso para la gestión de las organizaciones, cualquier cambio (incluso estructural) genera poca atención entre los usuarios de la información. Cuando se tiene en cuenta el tamaño de la organización se aprecia que las empresas grandes conocen más acerca de NIIF, mientras que en los otros tipos de empresa el conocimiento es nulo (García, Dueñas \& Moreno, 2011). La contabilidad ha estado y estará presente en los negocios y en las empresas, puesto que no es fácil medir impactos sin un conocimiento de la realidad económica y financiera. Esta disciplina ha servido de apoyo en la toma de decisiones para proyectar los entes económicos y ha evolucionado acorde con los cambios sociales (Valbuena, 2011).

El poder de la contabilidad se incrementó con el crecimiento de las economías de mercado en los distintos países y tendrá aún mayor importancia en la futura economía mundial. "La contabilidad constituye el órgano de vista de las empresas, debe permitir conocer, en cualquier momento, dónde se está y dónde se va, debe dar informes exactos, claros y precisos sobre la situación económica de la empresa"(Fayol, 1916, p.109).

Con la globalización de la economía, es necesario que los administradores de las industrias de la región y "organizaciones en general, posean una contabilidad basada en realidad económica y que además compita con la de los países con los que se espera existan intercambios económicos; esto se da a través de la implementación de NIIF (Janica \& Piñero, 2008). Así, la contabilidad generada bajo estándares internacionales presentará estados financieros con información de mejor calidad, comparable y coherente con los requerimientos de los usuarios, que se convierte en la obtención de ventaja competitiva en el momento de realizar intercambios económicos en mercados globalizados.

\subsection{Estrategias de mejoramiento de la intervención de la gerencia en el proceso de transición}

A partir de las consideraciones anteriores y aplicando un enfoque metodológico exploratorio analítico, toda vez que la temática objeto de estudio ha sido poco explorada a nivel local, se pueden derivar estrategias de mejoramiento de la intervención de la Administración de las organizaciones que permitan optimizar el proceso de transición a NIIF, en las PyMES de Sogamoso, evadiendo obstáculos experimentados en otros países, contribuyendo de esta manera a una conversión más efectiva. A continuación se relacionan algunas de estas estrategias:

La administración de las PyMES de Sogamoso debe ser consciente de la importancia de su intervención en el proceso de transición a NIIF, participación que debe iniciarse desde el principio de la actividad, de lo contrario este proceso no solo será más tedioso sino que los costos de la aplicación serán más altos. 
La gerencia de las PyMES de Sogamoso debe comenzar a planear desde ahora el proceso de conversión a NIIF, pues las compañías que inicien el proceso de transición de forma temprana se beneficiarán con tiempos de puesta en marcha más extensos, para abordar estos cambios con proactividad.

El proceso de transición a NIIF es un proyecto de alta complejidad y trascendencia para la organización, por lo tanto la administración de las PyMES de Sogamoso deben planear, estructurar y proveer los recursos necesarios que le permita gestionar de manera más eficiente esta labor.

La gerencia de las PyMES de Sogamoso debe iniciar un proceso de capacitación y entrenamiento por parte de personas expertas en el tema, dirigido al personal de las áreas funcionales impactadas por el proyecto donde participe igualmente la administración.

La administración de las PyMES en Sogamoso debe propender por la asignación de espacios físicos adecuados, que sin lugar a dudas contribuirán a que el objetivo de la convergencia se logre de mejor manera y de forma oportuna.

Los administradores de las PyMES en Sogamoso deben ser conscientes de los impactos que se verán reflejados en la gran mayoría de las áreas de la organización ya que, cualquier función del negocio requerida para preparar información financiera, o impactada por información financiera, tiene el potencial para ser afectada por el cambio.

La administración de las PyMES debe ser muy proactiva en el manejo de las comunicaciones tanto internas como externas, pues su mejoramiento puede llevar a que se conozcan las percepciones de las personas involucradas en el proceso, reduciendo el riesgo de malos entendidos que puedan incidir en el proceso de conversión a NIIF.

\section{Conclusiones}

A través del estudio realizado a lo largo de este escrito, se pueden deducir o reconocer las siguientes derivaciones:

Las PyMES en Sogamoso, al igual que en el ámbito mundial, se han consolidado como el foco de desarrollo económico de la región, por lo cual es necesario definir estrategias que les permitan mantenerse y ser competitivas en mercados nacionales e internacionales. Sin embargo, los empresarios no le brindan la importancia necesaria al proceso de transición a NIIF debido a que los cambios en el área contable les generan poca atención, ya que su interés está centrado en otras áreas funcionales del negocio. Por ello es importante la concientización e intervención de los administradores en dicha actividad, pues como ya se experimentó en otros países, si no existe una efectiva participación de la gerencia, el proceso de transición estará destinado al fracaso.

Las PyMES en Sogamoso cuentan con pocos recursos económicos, que son importantes en el momento de dirigir un proceso de transición a NIIF, por lo tanto la administración de estas organizaciones debe propender por la reducción de costos, esto se logra siendo proactivo iniciando este proyecto desde ya, permitiéndole a la administración gestionar los recursos con mayor eficiencia. Igualmente, la gerencia debe ser consciente de la obligatoriedad de la transición a NIIF y de los beneficios de reportar un conjunto completo de estados financieros con base en estándares contables internacionales, que facilitará el acceso a los mercados y la posible reducción de costos para las empresas y, por lo tanto, el mejoramiento de la competitividad global.

Los estados financieros presentados bajo estándares internacionales serán para los administradores de las PyMES de Sogamoso, una 
herramienta útil de control de gestión y un instrumento importante para empezar a planear estratégicamente su actuación en el mercado, teniendo en cuenta que en este momento no poseen dichos elementos para su gestión. En éste sentido se hace indispensable un acompañamiento por parte de las entidades que conforman la comunidad administrativa y contable en la región y en Sogamoso con capacitaciones que incluyan cursos, seminarios, diplomados y especializaciones que profundicen en el tema.

Al contrastar la teoría con la realidad de las organizaciones de la región, se sugiere la posibilidad de desarrollar una investigación con un fuerte contenido empírico que indague en las propias empresas acerca de las prácticas contables que históricamente han manejado, la utilización que hayan hecho de la información y las ventajas que podrían encontrar y desarrollar a partir de la convergencia a NIIF.

\section{Agradecimientos}

Este Artículo se presenta como opción de grado al título de Magíster en Administración, de la Universidad Nacional de Colombia; fue elaborado bajo la dirección de Phd. Zuray Andrea Melgarejo Molina, profesora asociada, Universidad Nacional de Colombia, a quien quiero agradecer por su acompañamiento, que hizo posible la elaboración de este manuscrito.

\section{Referencias}

Arango, L. \& López, C. (1999). Globalización, Apertura Económica $Y$ Relaciones Industriales en América Latina. Bogotá: Utópica Ediciones.

Ávila, F. (2013). Plan de responsabilidad social empresarial: una propuesta para pequeñas y medianas empresas industriales de la provincia de Tundama. Criterio Libre, 11(18), 217-228. Recuperado de http://www.unilibre.edu.co/Crite rioLibre/images/revistas/18/criterio-libre-9.pdf
Bassino, E. (2009, diciembre). Para una mejor información financiera global. Periódico Informativo de la Cámara Argentino-Danesa de Industria Comercio. Recuperado de http//cai.m debuenosaires.gov.ar/contenido/objetos/ revista.pdf

Bedodo, V. \& Giglio, C. (2006). Motivación laboral y compensaciones: una investigación de orientación teórica, Universidad de Chile.

Bermúdez, J. \& Gutiérrez, O. (2012). Las prácticas de innovación en las PyMES boyacenses. Apuntes delCENES, 31 (54), 161-192.

Bozkurt, O, Islamoglu M. \& OzY. (2013). Perceptions of professionals interested in accounting and auditing about acceptance and adaptation of global financial reporting standards. Journal of Economics, Finance and Administrative science, 18 (34), 16-23.

Burgos, H. (2007, noviembre). Normas Internacionales de Contabilidad. Revista Panorama. Internacionalización de Pymes, Recuperado de http://repository.poligran. edu.co/bitstream/10823/87/1/panorama3.pdf

Bustelo, P. (2005). La Nueva Arquitectura Financiera Internacional. Cuadernos De La Escuela Diplomática, (26), 153-158.

Calderón, G., Naranjo, J. \& Álvarez, C. (2007). La gestión humana en Colombia: características y tendencias de la práctica y de la investigación. Estudios Gerenciales, 23 (103), 39-64.

Cano, A. (2009). Las Normas Internacionales en la Contabilidad y la entrada en vigencia de la ley 1314 De 2009 en el contexto contable Colombiano. Observatorio de la Economía Latinoamericana, (121). Recuperado de http://www.eumed.net/cursecon/ecolat/co/09/a mcm.htm 
Carreño, A. (2012). Estados financieros tradicionales, una limitante en las decisiones gerenciales. (Tesis de Maestría, Universidad Nacional de Colombia, Sede Manizales), Recuperado de: http://www.bdigital.unal.edu.co/ 9259/1/7708506.2012.pdf

Carrera, J. (2009). El G20, la crisis y rediseño de la Arquitectura Financiera Internacional. Banco Central La República De Argentina BCRA, Ensayos Económicos, (53-54), 217, 225. Recuperado de http://www.bcra.gov.ar/pdfs/investigaciones/5354-10-Carrera.pdf

Castiglione, G. (2012). Nueva arquitectura financiera internacional, una respuesta desde América Latina a la crisis. Revista de Información y Debate (54), 33-37. Recuperado de http://www.re vistapueblos.org/? $p=11967$

Colegio de Peritos Mercantiles y Contadores Públicos de Honduras. (2011). Las Normas de Información Financiera (NIIF): Expectativas y Realidades. Recuperado de http://www.elcon tador.org.hn/uploads/media/NIIF_EXPECTATIVAS _Y_REALIDADES.pdf

Conferencia de las Naciones Unidas sobre Comercio y Desarrollo UNCTAD. (2005). Examen de cuestiones relativas a la aplicación práctica de Las Normas Internacionales de Información Financiera. Recuperado de http://unctad.org/ es/Docs/c2isard28_sp.pdf

Consejo Técnico de la Contaduría Pública (2011). Convergencia hacia las Normas Internacionales de Información Financiera y Aseguramiento de la información (NIIF). Recuperado de http://www.fe nalco.com.co/sites/default/files/guianiif.pdf

Consejo Técnico de la Contaduría Pública (2012a). Direccionamiento Estratégico del proceso de convergencia de las Normas de Contabilidad e Información Financiera y de Aseguramiento de la Información con Estándares Internacionales. Recuperado de http://www.globalcon table.com/attachments/studies/direcciona miento_05diciembre_2012.pdf

Consejo Técnico de la Contaduría Pública (2012b). La convergencia a IFRS lo que las juntas directivas y los comités de auditoría deberían saber y hacer. Recuperado de http://www.ctcp.gov.co/sites/ default/files/DtoResumenJuntasDirectivas6julio2 012Final.pdf

Contreras, L. (2010, 25 de febrero). NIIF para las PyMES. Global Contable. Recuperado de http://www.globalcontable.com/index.php?opti on=com_content\&view=article\&id=581:niifpara-las-pymes-en-chile\&catid=14:Art $\%$ C3\%83\% C2\%ADculos\&ltemid $=122$

Evia, M. (2006). ¿Es la Contabilidad Administrativa una Herramienta útil para desarrollar la competitividad en las empresas?. Contaduría y Administración, (219), 156-166.

Fayol, H. (1916). Administration industrielle et generalePrévoyance - Organisation Commandement - Coordination - Contrôle. Paris: Dunot.

Flórez, E. (2012, 4 de junio). Inclusión de todas las áreas de la empresa según NIIF [Audio podcast]. Recuperado de http://www.youtube.com/ watch? $\mathrm{v}=\mathrm{kdS}-63 \mathrm{coHUM}$

García, N., Dueñas, N. \& Moreno, J. (2011). Percepciones de las Organizaciones sobre la Contabilidad Financiera. Una ilustración para la ciudad de Bucaramanga - Colombia. Documentos FCE. Universidad Nacional de Colombia, (6), 1-26. Recuperado de http://www.bdigital.unal.edu.co/ 3451/1/docgarcia_eacp6.pdf

García, N. \& Sierra, E. (2012). Un análisis de la reforma contable en Colombia. Revista Internacional de LEGIS de Contabilidad Y Auditoría, (51), 38-54. Recuperado de http://www.academia. edu/2049786/Un_Analisis_de_la_Reforma_Cont able_en_Colombia 
Gil, J., (2013, 30 de Enero). Si se cumplen los pasos, la adopción de las NIIF llegará a feliz término. ambitojuridico.com. Recuperado de http://www. glenif.org/es/index.php?option=com_content\&vi ew $=$ article\&id=122:entrevista-con-el-vicepre sidente-jorge-jose-gil\&catid=36:noticias\& Itemid $=55$

González, J., Cerón, C. \& Alcázar, F. (2010). Caracterización emprendedora de los empresarios en los Valles de Tundama y Sugamuxi Boyacá (Colombia). Pensamiento \& Gestión, (29), 163-189.

González, J., Rodríguez, M. \& Duarte, L. (2011) Necesidades de consultoría en las MIPYMES del Valle de Sugamuxi en Boyacá. Cuadernos de Administración, 27 (46), 67-80.

Gutiérrez, O. \& Bermúdez, J. (2011). Estudio exploratorio sobre buenas prácticas de innovación en un grupo de pequeñas y medianas empresas (PyMES) en Boyacá. AD-minister, (19), 21 44.

Janica, F. \& Piñeros, J. (2008). USGAAP vs, IFRS y COLGAAP Lo Básico. Ernst \&Young Audit Ltda. Recuperado del sitio de internet http://www. globalcontable.com/archivosgenerales/seccione s/ifrs/comparativos/ey/colgaapbasico.pdf.

Ley 222 Capítulo IV Estados financieros. (1995). Recuperado de http://www.secretariasenado. gov.co/senado/basedoc/ley/1995/ley_0222_199 5.html

Londoño, J. (2011). Responsabilidad de los Administradores frente a las NIIF, Convergencia hacia las Normas Internacionales de Información Financiera Y aseguramiento de la Información (NIIF). Ministerio de Comercio de Industria y Turismo, 169. Recuperado de http://www.fenalco. com.co/sites/default/files/guianiif.pdf
Mallo, C. \& Pulido, A. (2005). Nuevo encuadre de la contabilidad para la dirección de la empresa (Management Accounting) ante la implantación global de las NIIF. Congresso Internacional de Custos Congreso llevado a cabo en el IX Congreso Internacional de Costos, Florianópolis, Brasil. (pp. 2-16)

Mantilla, S. A. (2012). Estándares/ Normas Internacionales de Información Financiera IFRS (NIIF). Bogotá, Colombia: Ecoe Ediciones.

Maticorena, W. (2013, 26 de junio). Las PyMES y su conversión a normas NIIF. Colombia.com, Recuperado de http://www.colombia.com/ actualidad/economia/sdi/65042/las-pymes-y-suconversion-a-las-normas-niif

Mejía, E., Montes, C. \& Montilla, O. (2008). Fundamentos teóricos del modelo contable común para las PyMES de América Latina: una alternativa a la regulación contable internacional IASB, Estudios Gerenciales, 24 (107), 59-85.

Mondragón, J. (2011). Innovación para la integración global de las PyMES. PyME la revista, Asociación de las Micro, Pequeñas y Medianas Empresas ACOPI, (25)

Quiroga, D. (2007). Comunicación, clima y cultura organizacional para la gestión del conocimiento. PyMES metalmecánicas de Cali. Revista UniversidadyEmpresa 6(13), 9-36.

Ramírez, H. \& Suárez, L. (2012). 131 casos sobre NIIF para PYMES. Bogotá D.C.- Cali: Normas Gerenciales-NIIF-IFRS-NIC-IAS-PYMES. Recuperado de http://elblogdegeovanirodriguez. bligoo.es/media/users/22/1125796/files/303797/ 131-casos-niif-ebook.pdf

Ramos, G. (2001, 26 de marzo) La Nueva Arquitectura Financiera Internacional. Boletín económico de ICE, (2686), 19-38. 
Rodeiro D. \& López, M. (2007). La innovación como factor clave en la competitividad empresarial: un estudio empírico en PyMES. Revista Galega de Economía, 16(2), 1-18.

Rodríguez, J. (2008). Fuentes de Información para las PyME, en el ámbito internacional y Nacional, de Organismos Gubernamentales o privados, y por los medios de comunicación. Gestión y estrategia, (33), 47-61.

Saizarbitoria, I. H. \& Casadesús, M., (2006) Los estándares internacionales de sistemas de gestión Pasado, presente y futuro. Revista Información Comercial Española, Boletín Económico, (2676), 4561.

Superintendencia de sociedades (2012). Circular externa 115-000002. Recuperado de http://www. supersociedades.gov.co/imagenes/cominicacion es/2012-01-048021-000.pdf

Valbuena, J. (2011). El contador internacional, una nueva dimensión del contador público externadista. Apuntes Contables, (15), 91-109. Recuperado de http://revistas.uexternado.edu. co/index.php/contad/article/view/2940/2584
Varón, L. (2012). Nueva Arquitectura Financiera Internacional. Recuperado de http://www.google. com.co/webhp?source=search_app\&gws_rd=cr\& ei=CU8xUuLvEou69gSb0YHQCQ\#q=Nueva+Arqu itectura+Financiera+Internaciona

Vélez, M. (2009). Desarrollo de un micromundo orientado a soportar la Administración de crecimiento en pequeñas y medianas Empresas. Universidad Nacional de Colombia.

Wyman, H. (1990). El poder y la importancia de la Contabilidad. Revista Española de Financiación y Contabilidad, 20 (62), 9-25.

Yandar, S. (2011). La dinámica del proceso de innovación en PyMES. El caso de dos empresas del sector agroindustrial. (Tesis de Maestría, Universidad Nacional de Colombia). Recuperado de:http://www.bdigital.unal.edu.co/4139/

Zapata, E. E. (2011). La efectividad del mercadeo de las PyMES en Boyacá. Apuntes del cenes, 20 (31), 245-260.

Zambrano, S. (2012). La competitividad en los sectores manufacturero y de servicios. Estudio de caso. Criterio Libre, 10 (17), 177-192. 